\title{
Arm Name
}

National Cancer Institute

\section{Source}

National Cancer Institute. Arm Name. NCI Thesaurus. Code C93729.

A non-unique textual identifier for the arm. 